\title{
A NOTE ON EQUIVARIANT NORMAL FORMS OF POISSON STRUCTURES
}

\author{
Eva Miranda And Nguyen Tien Zung
}

\begin{abstract}
We prove an equivariant version of the local splitting theorem for tame Poisson structures and Poisson actions of compact Lie groups. As a consequence, we obtain an equivariant linearization result for Poisson structures whose transverse structure has semisimple linear part of compact type.
\end{abstract}

\section{Introduction}

The main purpose of this note is to prove an equivariant version of Weinstein's splitting theorem for Poisson structures [17]. This theorem asserts that in the neighborhood of any point $p$ in a Poisson manifold $\left(P^{n}, \Pi\right)$ there is a local coordinate system $\left(x_{1}, y_{1}, \ldots, x_{k}, y_{k}, z_{1}, \ldots, z_{n-2 k}\right)$ in which the Poisson structure $\Pi$ can be written as

$$
\Pi=\sum_{i=1}^{k} \frac{\partial}{\partial x_{i}} \wedge \frac{\partial}{\partial y_{i}}+\sum_{i j} f_{i j}(z) \frac{\partial}{\partial z_{i}} \wedge \frac{\partial}{\partial z_{j}},
$$

where $2 k$ is the rank of $\Pi$ at $p$, and $f_{i j}$ are functions which depend only on the variables $\left(z_{1}, \ldots, z_{n-2 k}\right)$ and which vanish at the origin. Geometrically speaking, locally the Poisson manifold $\left(P^{n}, \Pi\right)$ can be splitted into the direct product of a $2 k$ dimensional symplectic manifold (with the standard nondegenerate Poisson structure $\left.\Pi_{1}=\sum_{i=1}^{k} \frac{\partial}{\partial x_{i}} \wedge \frac{\partial}{\partial y_{i}}\right)$ and a $(n-2 k)$-dimensional Poisson manifold whose Poisson structure $\Pi_{2}=\sum_{i j} f_{i j}(z) \frac{\partial}{\partial z_{i}} \wedge \frac{\partial}{\partial z_{j}}$ vanishes at the origin. We want to show that if there is a (local) action of a compact Lie group $G$ on $P^{n}$ which fixes the point $p$ and which preserves $\Pi$, then this splitting can be made equivariantly.

In the special case when $\Pi$ is nondegenerate at $p$ (i.e., $2 k=n$ ), one recovers from Weinstein's theorem the classical Darboux theorem about the local existence of canonical (Darboux) coordinates for symplectic manifolds. We know two methods for proving Darboux theorem: 1) the classical coordinate-by-coordinate construction method; and 2) the path method due to Moser [11]. Weinstein's proof of the splitting theorem [17] is also based on the first method (coordinate by coordinate construction). However, this classical method does not seem to work in the equivariant situation, while the path method can be used to prove the equivariant Darboux theorem [16].

Received by the editors 16 March, 2006.

1991 Mathematics Subject Classification. 53D17.

Key words and phrases. Poisson manifolds; Linearization; Normal Forms.

The first author is supported by Marie Curie EIF postdoctoral fellowship contract number EIF2005-024513 and partially supported by the DGICYT project number BFM2003-03458. 
In the same spirit, we will try to use the path method to prove an equivariant version of the splitting theorem for Poisson structures. In doing so, we encounter a technical condition, which we call the tameness condition: a smooth Poisson structure $\Pi$ on a manifold $P^{n}$ is called tame if for any two smooth Poisson vector fields $X, Y$ on $P^{n}$ (which may depend on some parameters) which are tangent to the symplectic leaves the function $\Pi^{-1}(X, Y)$ is smooth (and depends smoothly on the parameters). We will devote Section 2 of this note to the tameness condition, in order to convince the reader that it is an interesting condition, and many "reasonable" Poisson structures satisfy it. For example, if the linear part of the transverse Poisson structure at a point $p$ has semisimple type, then the Poisson structure is tame near $p$.

Now we can formulate the main result of this note:

Theorem 1.1. Let $\left(P^{n}, \Pi\right)$ be a smooth Poisson manifold, $p$ a point of $P, 2 k=$ rank $\Pi(p)$, and $G$ a compact Lie group which acts on $P$ in such a way that the action preserves $\Pi$ and fixes the point $p$. Assume that the Poisson structure $\Pi$ is tame at $p$. Then there is a smooth canonical local coordinate system $\left(x_{1}, y_{1}, \ldots, x_{k}, y_{k}\right.$, $\left.z_{1}, \ldots, z_{n-2 k}\right)$ near $p$, in which the Poisson structure $\Pi$ can be written as

$$
\Pi=\sum_{i=1}^{k} \frac{\partial}{\partial x_{i}} \wedge \frac{\partial}{\partial y_{i}}+\sum_{i j} f_{i j}(z) \frac{\partial}{\partial z_{i}} \wedge \frac{\partial}{\partial z_{j}},
$$

and in which the action of $G$ is linear and preserves the subspaces $\left\{x_{1}=y_{1}=\ldots x_{k}=\right.$ $\left.y_{k}=0\right\}$ and $\left\{z_{1}=\ldots=z_{n-2 k}=0\right\}$.

Remark 1.2. i) We do not know whether the tameness condition is really necessary, or if it is because our method is not good enough. We notice that this condition is also implicitly present in the papers of Ginzburg and Weinstein [8] and of Aleekseev and Meinrenken [1], [2], which involve the path method in Poisson geometry.

ii) The above theorem also holds in the analytic (i.e., real analytic or holomorphic) setting, with basically the same proof. The analytic version of this equivariant theorem is used by Philippe Monnier and the second author in their study of normal forms of vector fields on Poisson manifolds [10]. We hope that our result can be useful in the study of equivariant Hamiltonian systems as well.

iii) If the action of $G$ on $\left(P^{n}, \Pi\right)$ is Hamiltonian (with an equivariant momentum map), then there is another approach to this equivariant splitting problem, based on the Nash-Moser method, which does not need the tameness condition. We will consider this issue in a separate work.

The above theorem will be proved in Section 3 of this note. In Section 4 we will combine this theorem with linearization results of Conn [4] and Ginzburg [7] to obtain an equivariant linearization theorem (see Theorem 4.1).

\section{Tame Poisson structures}

We will denote by $\Pi^{-1}$ the covariant tensor dual to the Poisson tensor $\Pi$ of a Poisson manifold $\left(P^{n}, \Pi\right)$, i.e. the symplectic form on symplectic leaves. If $X, Y$ are vector fields on $P^{n}$ which are tangent to the symplectic leaves, then $\Pi^{-1}(X, Y)$ is well-defined. In particular, if $X=X_{h}$ is the Hamiltonian vector field of a function $h$ 
on $\left(P^{n}, \Pi\right)$ then $\Pi^{-1}(X, Y)=-Y(h)$. Recall that a Poisson vector field is a vector field which preserves the Poisson structure.

Definition 2.1. Let $\left(P^{n}, \Pi\right)$ be a smooth Poisson manifold and $p$ a point in $P$. We will say that $\Pi$ is tame at $p$ if for any pair $X_{t}, Y_{t}$ of germs of smooth Poisson vector fields near $p$ which are tangent to the symplectic foliation of $\left(P^{n}, \Pi\right)$ and which may depend smoothly on a (multi-dimensional) parameter $t$, then the function $\Pi^{-1}\left(X_{t}, Y_{t}\right)$ is smooth and depends smoothly on $t$.

The tameness condition is a kind of homological condition. In particular, if the parametrized germified first Poisson cohomology group, which we will denote by $H_{\Pi}^{1}\left(P^{n}, p\right)$, vanishes, then $\Pi$ is tame at $p$. Indeed, $H_{\Pi}^{1}\left(P^{n}, p\right)=0$ means that if $X_{t}$ is a germ of Poisson vector field near $p$ which depends smoothly on a parameter $t$, then we can write $X_{t}=X_{h_{t}}$ where $h_{t}$ is a germ of smooth function near $p$ which depends smoothly on the parameter $t$. Hence $\Pi^{-1}\left(X_{t}, Y_{t}\right)=-Y_{t}\left(h_{t}\right)$ is smooth.

In particular, it is known that if $\mathfrak{g}$ is a compact semi-simple Lie algebra, and $\left(\mathfrak{g}^{*}, \Pi_{\text {lin }}\right)$ is the dual of $\mathfrak{g}$ equipped with the corresponding linear Poisson structure then $H_{\Pi_{l i n}}^{1}\left(\mathfrak{g}^{*}, 0\right)=0$ (see [4]). Hence our first example of tame Poisson structures:

Example 2.2. Any smooth Poisson structure $\Pi$, which vanishes at a point $p$ and whose linear part at $p$ corresponds to a compact semisimple Lie algebra $\mathfrak{g}$, is tame at $p$. Indeed, in this case, according to Conn's smooth linearization theorem $[4],\left(P^{n}, \Pi\right)$ is locally isomorphic near $p$ to $\left(\mathfrak{g}^{*}, \Pi_{l i n}\right)$, and therefore $H_{\Pi}^{1}\left(P^{n}, p\right)=0$.

If $X$ is not Hamiltonian (and maybe not even Poisson) but can be written as $X=\sum_{i=1}^{m} f_{i} X_{g_{i}}$ where $f_{i}, g_{i}$ are smooth functions, then $\Pi^{-1}(X, Y)=-\sum_{i=1}^{m} f_{i} Y\left(g_{i}\right)$ is still smooth. This leads us to:

Definition 2.3. We say that a smooth (resp real analytic) Poisson structure $\Pi$ satisfies the smooth division property (resp analytic division property) at a point $p$ if the Hamiltonian vector fields generate the space of vector fields tangent to the associated symplectic foliation near $p$. More precisely, for any germ of smooth (resp. analytic) vector field $Z$-which may depend smoothly (resp. analytically) on some parameterswhich is tangent to the symplectic foliation there exists a finite number of germs of smooth (resp. analytic) functions $f_{1}, \ldots, f_{m}, g_{1}, \ldots, g_{m}$ - which depend smoothly (resp. analytically) on the same parameters as $Z$ - such that $Z=\sum f_{i} X_{g_{i}}$.

Clearly, if $\Pi$ satisfies the division property at a point $p$, then it is tame at $p$. A natural question is to know which Poisson structures satisfy the division property. In particular, is it true that all linear Poisson structures satisfy the division property at the origin? In the appendix we prove that low-dimensional Lie algebras satisfy the division property at the origin. Namely

Proposition 2.4. Any linear Poisson structure in dimension 2 or 3 has the division property at the origin.

In the higher-dimensional case, a result of Dixmier [5] says (in our language) that if $\Pi$ is a linear Poisson structure which corresponds to a semisimple Lie algebra then it has the analytic division property at the origin (mainly due to the fact that the singular set has codimension 3 in this case). We would conjecture that Dixmier's 
result also holds in the smooth case. On the other hand, one can probably produce linear Poisson (non semisimple) structures which do not satisfy the division property (similiar to Dixmier's counterexample 3.3 in [5]).

It is not difficult to construct examples of Poisson structures with a trivial 1-jet which are not tame.

Example 2.5. Consider the Poisson structure $\Pi=x^{4} \frac{\partial}{\partial x} \wedge \frac{\partial}{\partial y}$ on $\mathbb{R}^{2}$. The following vector fields are Poisson and tangent to the symplectic foliation:

$$
X=x^{2} \frac{\partial}{\partial x}+2 x y \frac{\partial}{\partial y}, \quad Y=x \frac{\partial}{\partial y},
$$

but $\Pi^{-1}(X, Y)=\frac{1}{x}$ is not smooth at the origin. So this Poisson structure is not tame.

Recall that if $\Pi=\sum_{i=1}^{k} \frac{\partial}{\partial x_{i}} \wedge \frac{\partial}{\partial y_{i}}+\sum_{i j} f_{i j}(z) \frac{\partial}{\partial z_{i}} \wedge \frac{\partial}{\partial z_{j}}$ in a local canonical coordinate system in the neighborhood of a point $p$, then $\Pi_{2}=\sum_{i j} f_{i j}(z) \frac{\partial}{\partial z_{i}} \wedge \frac{\partial}{\partial z_{j}}$ is called the transverse Poisson structure of $\Pi$ at $p$. Up to local Poisson isomorphisms, this Poisson transverse structure is unique, i.e. it does not depend on the choice of local canonical coordinates, see, e.g., $[6,17]$. The following lemma shows that, to verify the tameness condition, it is sufficient to check it in the transverse direction to the symplectic leaf:

Lemma 2.6. A smooth Poisson structure $\Pi$ is tame at a point $p$ if and only if the transverse Poisson structure of $\Pi$ at $p$ is tame at $p$.

Proof. Write $\Pi=\Pi_{1}+\Pi_{2}=\sum_{i=1}^{k} \frac{\partial}{\partial x_{i}} \wedge \frac{\partial}{\partial y_{i}}+\sum_{i j} f_{i j}(z) \frac{\partial}{\partial z_{i}} \wedge \frac{\partial}{\partial z_{j}}$ in a local canonical coordinate system near $p$. For each germ of vector field $X$ near $p$ write $X=X_{\text {hor }}+$ $X_{\text {vert }}$, where $X_{h o r}$ is the "horizontal part" of $X$, i.e. is a combination of the vector fields $\frac{\partial}{\partial x_{i}}, \frac{\partial}{\partial y_{i}}$, and $X_{\text {vert }}$ is the "vertical part" of $X$, i.e. is a combination of the vector fields $\frac{\partial}{\partial z_{i}}$. If $X$ is a smooth Poisson vector field for $\Pi$, then $X_{h o r}$ (resp. $X_{\text {vert }}$ ) may be viewed as a Poisson vector field for $\Pi_{1}$ (resp., $\Pi_{2}$ ) which depends smoothly on parameters $z_{i}$ (resp., $\left.x_{i}, y_{i}\right)$. We have $\Pi^{-1}(X, Y)=\Pi_{1}^{-1}\left(X_{h o r}, Y_{h o r}\right)+$ $\Pi_{2}^{-1}\left(X_{\text {vert }}, Y_{\text {vert }}\right)$. The term $\Pi_{1}^{-1}\left(X_{\text {hor }}, Y_{\text {hor }}\right)$ is always smooth (provided that $X$ and $Y$ are smooth), and so the smoothness of $\Pi^{-1}(X, Y)$ is equivalent to the smoothness of $\Pi_{2}^{-1}\left(X_{\text {vert }}, Y_{\text {vert }}\right)$. The lemma then follows easily.

\section{Proof of the equivariant splitting theorem}

In this section we will give a proof of Theorem 1.1. It uses coupling tensors for Poisson manifolds, so we will first recall a result of Yu. Vorobiev about coupling tensors (see, e.g., $[6,14]$ ). The proof of the theorem consists of three steps. In the first step we prove that we can assume that the action of our compact Lie group $G$ is linear and that the symplectic foliation is normalized (i.e. is the same as in the splitting theorem). In the second step we construct a path of $G$-invariant Poisson structures connecting the initial Poisson structure to the splitted one. Finally, in the last step, we use this path of Poisson structures and the averaging method to construct a flow which intertwines with the action of $G$ and whose time-1 map moves 
the initial Poisson structure to the splitted one, thus giving an equivariant splitting of our Poisson structure.

\subsection{Preliminaries: coupling tensors.}

Let $\pi: E \longrightarrow S$ be a submersion over a manifold $S$ and let $T_{V} E=\operatorname{ker} d \pi$. An Ehresmann connection on $E$ is a splitting of the tangent bundle of $E$ as $T E=T_{V} E \oplus$ $T_{H} E$. We call $T_{H} E$ the horizontal space. Denote by $\mathcal{V}_{V}^{1}(E)$ the set of vertical vector fields. We can associate to this splitting a $\mathcal{V}_{V}^{1}(E)$-valued 1-form $\Gamma \in \Omega^{1}(E) \otimes \mathcal{V}_{V}^{1}(E)$ such that $\Gamma(Z)=Z$ for any vertical vector field. Then the horizontal space can be written as $T_{H} E=\{X \in T E, \quad \Gamma(X)=0\}$. We can define the horizontal lifting of vector fields from $S$ to $E$. In the same way, we may associate a parallel transport to $\Gamma$ which is smooth, a curvature form and a covariant derivative (for details see for example $[6])$.

Consider now the case when $S$ is a symplectic leaf of a Poisson manifold $(P, \Pi)$. We can consider a neighbourhood $E$ of $S$ and submersion $\pi: E \longrightarrow S$ whose restriction to $S$ is the identity.

There is a natural smooth Ehresmann connection where the horizontal subbundle is spanned by the Hamiltonian vector fields $X_{f \circ \pi}$.

We can also associate to it a 2 -form $\mathbb{F} \in \Omega^{2}(S) \otimes \mathcal{C}^{\infty}(E)$ defined as

$$
\mathbb{F}\left(X_{f \circ \pi}, X_{g \circ \pi}\right)=\left\langle\Pi, \pi^{*} d f \wedge \pi^{*} d g\right\rangle .
$$

Recall that we have an induced transverse Poisson structure $\Pi_{V e r t}$ on the vertical space.

The triple $\left(\Pi_{V e r t}, \Gamma, \mathbb{F}\right)$ is called the geometric data associated to the Poisson manifold $(P, \Pi)$ in a neighbourhood of a symplectic leaf. In [14], Vorobjev studies the reconstruction problem from given geometric data. That is, given a triple of smooth geometric data he gives compatibility conditions that guarantee the existence of a Poisson structure with those geometric data. Those compatibility conditions come from the Schouten condition $[\Pi, \Pi]=0$ imposed on the bivector field $\Pi$ reconstructed from the geometric data.

Assume that we are given $\left(\Pi_{V e r t}, \Gamma, \mathbb{F}\right)$ on a fibration $\pi: E \longrightarrow S$, where $\Gamma$ is an Ehresmann connection on $E, \Pi_{V e r t}$ a vertical bivector field, and $\mathbb{F} \in \Omega^{2}(S) \otimes \mathcal{C}^{\infty}(E)$ a nondegenerate $\mathcal{C}^{\infty}(E)$-valued 2-form on $S$.

We will need the following characterization of geometrical data which come from a Poisson structure:

Theorem 3.1 (Vorobiev [14]). The triple $\left(\Pi_{V e r t}, \Gamma, \mathbb{F}\right)$ on a fibration $\pi: E \longrightarrow$ $S$ determines a Poisson structure on $E$ if and only if $\mathbb{F}$ is nondegenerate and the following four compatibility conditions are satisfied:

$$
\begin{aligned}
& {\left[\Pi_{V e r t}, \Pi_{V e r t}\right]=0,} \\
& L_{H o r(u)}\left(\Pi_{V e r t}\right)=0 \quad \forall u \in \mathcal{V}_{V}^{1}(E), \\
& \partial_{\Gamma} \mathbb{F}=0, \\
& \operatorname{Curv}_{\Gamma}(u, v)=\nu^{\sharp}(d(\mathbb{F}(u, v))),
\end{aligned}
$$


where $\partial_{\Gamma}$ stands for the covariant derivative and $\nu^{\sharp}$ stands for the map from $T^{*} E$ to TE defined by $\left\langle\nu^{\sharp}(\alpha), \beta\right\rangle=\langle\nu, \alpha \wedge \beta\rangle$.

Remark 3.2. We may think of $\Pi$ as the coupling of $\Pi_{V e r t}$ with $\mathbb{F}$ by $\Gamma$. This so-called coupling method is a generalization of the minimal coupling procedure established for symplectic fibrations by Guillemin, Lerman and Sternberg [9], [13].

\subsection{First step of the proof: linearization of the group action.}

Consider an action $\rho: G \times P^{n} \rightarrow P^{n}$ of a compact Lie group $G$ on a Poisson manifold $\left(P^{n}, \Pi\right)$, which fixes a point $p \in P^{n}$ and preserves the Poisson structure $\Pi$. Denote by $S$ the local symplectic leaf through $p$. Note that $S$ is invariant under the action of $G$. According to Bochner's theorem [3], the action of $G$ is linearizable near $p$, i.e., there is a local coordinate system in which the action is linear. Moreover, we may assume that $S$ is linear in these cordinates. Since linear representations of compact Lie groups are completely reducible, there is a local submanifold $N$ (which is also linear in these coordinates), which is invariant under the action of $G$ and which is transverse to $S$ at $p$. The following lemma says that we can choose this coordinate system in such a way that the symplectic foliation of $\left(P^{n}, \Pi\right)$ will also be the same as in the splitting theorem.

Lemma 3.3. With the above notations, there is a local system of coordinates near $p$ in which the action of $G$ is linear, the submanifolds $S, N$ are linear, and the local symplectic leaves near $p$ are direct products of $S$ with symplectic leaves of the transverse Poisson structure on $N$.

Proof. We can start with a first coordinate system in which the action of $G$ is linear and the submanifolds $S, N$ are linear. Denote by $p_{1}$ the linear projection from a sufficiently small neighborhood $U$ of $p$ in $P^{n}$ to $S$ which projects $N$ to $p$. Define another (a-priori nonlinear) projection $p_{2}$, from $U$ to $N$, as follows: Denote by $\Gamma$ the Ehresmann connection associated to the Poisson structure $\Pi$ and the projection $p_{1}$. For each $x \in U$, let $\alpha_{x}(t)$ be the linear path joining $p_{1}(x)$ to the origin $p$ in $S$, with $\alpha_{x}(0)=p_{1}(x)$ and $\alpha_{x}(1)=p$. Denote by $\hat{\alpha}_{x}$ the horizontal lift of $\alpha_{x}$ through $x$ with respect to $\Gamma$. Then we take $p_{2}(x)=\hat{\alpha}_{x}(1) \in N$.

By construction both projections are smooth and $G$-equivariant: The projection $p_{1}$ is equivariant since $N$ is $G$-invariant and $p_{2}$ is equivariant because the action of $G$ preserves $\Pi$ and therefore the parallel transport is equivariant.

Now consider the $G$-equivariant local diffeomorphism

$$
\begin{array}{ccc}
\phi: \quad U & \longrightarrow \times N \\
& x \longmapsto & \left(p_{1}(x), p_{2}(x)\right)
\end{array}
$$

Since the parallel transport preserves the Poisson structure, $\phi$ takes the Poisson structure on $U$ to a Poisson structure on $S \times N$ which has as symplectic leaves the product of the symplectic leaves on $N$ with $S$. This ends the proof of the lemma.

\subsection{Second step: constructing a path of Poisson structures.}

After the first step, we can now assume that $P=N \times S$, and the Poisson structure $\Pi$ has the same symplectic leaves as the splitted Poisson structure $\tilde{\Pi}=\Pi_{S}+\Pi_{N}$, where $\Pi_{S}$ is the standard nondegenerate Poisson structure on $S$ and $\Pi_{N}$ is the transverse 
Poisson structure on $N$, and both $\Pi$ and $\tilde{\Pi}$ are invariant under our linear action of $G$. We will assume that $\Pi$ is tame at $p$, or equivalently, the transverse Poisson structure $\Pi_{N}$ is tame at the origin.

Lemma 3.4. With the above notations and assumptions, there is a smooth path of $G$-invariant Poisson structures $\Pi_{t}, t \in[0,1]$, on (a neighborhood of the origin in) $N \times S$, such that $\Pi_{0}=\Pi, \Pi_{1}=\Pi_{S}+\Pi_{N}$, and which have the same symplectic foliation for all $t \in[0,1]$.

Proof. We denote by $\omega_{0}$ the symplectic structure induced on the symplectic leaves by $\Pi_{0}=\Pi$. In the same way we denote by $\omega_{1}$ the symplectic structure induced by $\Pi_{1}=\Pi_{S}+\Pi_{N}$ on the same symplectic foliation. Consider the linear path of 2 -forms

$$
\omega_{t}=t \omega_{1}+(1-t) \omega_{0} .
$$

This is a path of smooth closed 2-forms on each symplectic leaf of the common symplectic foliation. We want to show that, for each $t$ there is a smooth bivector field $\Pi_{t}$ which corresponds to $\omega_{t}$. Then, automatically, $\Pi_{t}$ is a Poisson structure because of the closedness of $\omega_{t}$, has the same symplectic foliation as $\Pi_{0}$ and $\Pi_{1}$, and is $G$-invariant.

Denote by $\left(\Pi_{N}, \Gamma_{0}, \mathbb{F}_{0}\right)$ and $\left(\Pi_{N}, \Gamma_{1}, \mathbb{F}_{1}\right)$, the geometric data associated to the Poisson structures $\Pi_{0}=\Pi$ and $\Pi_{1}=\Pi_{N}+\Pi_{S}$ with respect to the projection $p_{1}$ : $N \times S \rightarrow S$ (remark that, by construction, they have the same vertical component, which is equal to $\Pi_{N}$ ). We will use Vorobjev's Theorem 3.1 to construct $\Pi_{t}$ and to prove its smoothness. In other words, we will construct geometric data $\left(\Pi_{N}, \Gamma_{t}, \mathbb{F}_{t}\right)$, which will be shown to be smooth and satisfy the compatibility conditions of Theorem 3.1 , so they will give rise to a smooth Poisson structure $\Pi_{t}$.

\section{Construction and smoothness of $\Gamma_{t}$ :}

In order to construct the connection $\Gamma_{t}$, it is enough to show how to lift each vector field $X$ on $S$ horizontally with respect to $\Gamma_{t}$. The horizontal lift $X_{t}$ of $X$ with respect to $\Gamma_{t}$ is uniquely characterized by $\omega_{t}$ (the would-be associated symplectic form on the symplectic leaves) and by the following two conditions:

(1) The vector field $X_{t}$ is tangent to the common symplectic foliation of $\Pi_{0}$ and $\Pi_{1}$, and its projection to $S$ by $p_{1}$ is $X$.

(2) $\omega_{t}\left(X_{t}, Z\right)=0$ for any vertical vector field $Z$.

Denote by $X_{0}$ and $X_{1}$ the horizontal lift of $X$ with respect to $\Gamma_{0}$ and $\Gamma_{1}$ respectively. We will show that

$$
X_{t}=(1-t) X_{0}+t X_{1} .
$$

(Then the smoothness of $X_{t}$, and hence of $\Gamma_{t}$, is automatic). It is clear that (1t) $X_{0}+t X_{1}$ is tangent to the symplectic foliation and projects to $X$ under $p_{1}$. It remains to show that

$$
\omega_{t}\left((1-t) X_{0}+t X_{1}, Z\right)=0
$$


for any vertical vector field $Z$ on $N \times S$. Indeed, denoting $W=X_{0}-X_{1}$, we have

$$
\begin{aligned}
& \omega_{t}\left((1-t) X_{0}+t X_{1}, Z\right) \\
= & t \omega_{1}\left((1-t) X_{0}+t X_{1}, Z\right)+(1-t) \omega_{0}\left((1-t) X_{0}+t X_{1}, Z\right) \\
= & t \omega_{1}\left(X_{1}+(1-t) W, Z\right)+(1-t) \omega_{0}\left(X_{0}-t W, Z\right) \\
= & t \omega_{1}\left(X_{1}, Z\right)+(1-t) \omega_{0}\left(X_{0}, Z\right)+t(1-t)\left[\omega_{1}(W, Z)-\omega_{0}(W, Z)\right] .
\end{aligned}
$$

Since $X_{1}$ and $X_{0}$ are the horizontal lifts of $X$ with respect to $\Gamma_{1}$ and $\Gamma_{0}$, the terms $\omega_{1}\left(X_{1}, Z\right)$ and $\omega_{0}\left(X_{0}, Z\right)$ vanish. Since the Poisson structures $\Pi_{0}$ and $\Pi_{1}$ have the same transverse component, and $W$ and $Z$ are vertical vector fields, we have $\omega_{1}(W, Z)=\omega_{0}(W, Z)=\Pi_{N}^{-1}(W, Z)$. Hence $\omega_{t}\left((1-t) X_{0}+t X_{1}, Z\right)=0$ as desired.

Construction and smoothness of $\mathbb{F}_{t}$ :

If $X$ is a vector field on $S$ then we will denote by $X_{t}=(1-t) X_{0}+t X_{1}$ the horizontal lift of $X$ to $N \times S$ via $\Gamma_{t}$ as above. For any two smooth vector fields $X, Y$ on $S$ and a point $q \in N \times S$, put

$$
\mathbb{F}_{t}(X, Y)(q)=\omega_{t}\left(X_{t}, Y_{t}\right)(q)
$$

The main point here is to check the smoothness of the function $\mathbb{F}_{t}(X, Y)$ defined by the above formula, in a neighborhood of the origin in $N \times S$. Denote $Z^{X}=X_{0}-X_{1}$ and $Z^{Y}=Y_{0}-Y_{1}$; they are vertical vector fields. Since the Ehresmann connection $\Gamma_{i}(i=0,1)$ preserves the transverse Poisson structures, the vector fields $\hat{X}_{i}$ and $\hat{Y}_{i}$ preserve the transverse Poisson structure $\Pi_{N}$. Therefore the vertical vector fields $Z^{X}$ and $Z^{Y}$ also preserve the transverse Poisson structure. (They may be viewed as Poisson fields on $\left(N, \Pi_{N}\right)$ parametrized by $\left.S\right)$.

We can write $X_{t}=X_{0}-t Z^{X}=X_{1}+(1-t) Z^{X}$ and $Y_{t}=Y_{0}-t Z^{Y}=Y_{1}+(1-t) Z^{Y}$. Recall that if $X_{t}$ is horizontal with respect to $\Gamma_{t}$ and $Z$ is vertical then $\omega_{t}\left(X_{t}, Z\right)=0$. We have:

$$
\begin{aligned}
& \mathbb{F}_{t}(X, Y) \\
= & t \omega_{1}\left(X_{1}+(1-t) Z^{X}, Y_{1}+(1-t) Z^{Y}\right)+(1-t) \omega_{0}\left(X_{0}-t Z^{X}, Y_{0}-t Z^{Y}\right) \\
= & t \omega_{1}\left(X_{1}, Y_{1}\right)+(1-t) \omega_{0}\left(X_{0}, Y_{0}\right)+ \\
& +t(1-t)^{2} \omega_{1}\left(Z^{X}, Z^{Y}\right)+t^{2}(1-t) \omega_{0}\left(Z^{X}, Z^{Y}\right) \\
= & t \omega_{1}\left(X_{1}, Y_{1}\right)+(1-t) \omega_{0}\left(X_{0}, Y_{0}\right)+t(1-t) \Pi_{N}^{-1}\left(Z^{X}, Z^{Y}\right)
\end{aligned}
$$

By our tameness hypothesis, $\Pi_{N}^{-1}\left(Z^{X}, Z^{Y}\right)$ is smooth, and so $\mathbb{F}_{t}(X, Y)$ is smooth (and depends smothly on $t$ ).

Remark that $\mathbb{F}_{t}$ coincides with $\mathbb{F}_{0}$ and $\mathbb{F}_{1}$ at the origin $p$. Since $\mathbb{F}_{0}$ is nodegenerate, $\mathbb{F}_{t}$ is also nondegenerate in a neighborhood of $p$ in $N \times S$.

Since the form $\omega_{t}$ used in the construction of $\left(\Pi_{N}, \Gamma_{t}, \mathbb{F}_{t}\right)$ is closed on each symplectic leaf, the four compatibility conditions for the triple $\left(\Pi_{N}, \Gamma_{t}, \mathbb{F}_{t}\right)$ are automatically satisfied. Hence the triple $\left(\Pi_{N}, \Gamma_{t}, \mathbb{F}_{t}\right)$ corresponds to a smooth Poisson structure $\Pi_{t}$ in a neighborhood of $p$ in $N \times S$.

Moreover, by construction, $\Pi_{0}=\Pi, \Pi_{1}=\Pi_{N}+\Pi_{S}$, and $\Pi_{t}$ depends smoothly on $t$. Lemma 3.4 is proved. 


\subsection{End of the proof.}

According to Lemma 3.4, we now have a smooth path of $G$-invariant Poisson structures $\Pi_{t}$, where $\Pi_{0}$ is our initial Poisson structure, and $\Pi_{1}=\Pi_{N}+\Pi_{S}$ is the splitted one. (The action of $G$ is already linearized, and by the equivariant Darboux theorem we may assume that $\Pi_{S}$ is already equivariantly normalized, i.e. has Darboux form). In order to finish the proof of the theorem, it suffices to find a local diffeomorphism of $N \times S$ which commutes with the action of $G$ and which moves $\Pi_{0}$ to $\Pi_{1}$.

According to Weinstein's splitting theorem (or rather its parametrized version, whose proof is the same), there is a smooth family of local diffeomorphisms $\phi_{t}, t \in[0,1]$ such that $\phi_{t *}\left(\Pi_{0}\right)=\Pi_{t}$ and $\phi_{0}=I d$. Note that, a-priori, $\phi_{t}$ does not commute with the action of $G$. Denote by $X_{t}$ the time-dependent vector field whose flow generates $\phi_{t}$, i.e.,

$$
X_{t}\left(\phi_{t}(q)\right)=\frac{\partial \phi_{t}}{\partial t}(q)
$$

By derivation of the condition

$$
\phi_{t *}\left(\Pi_{0}\right)=\Pi_{t}
$$

we get the following equation for $X_{t}$ :

$$
L_{X_{t}}\left(\Pi_{t}\right)=-\frac{d \Pi_{t}}{d t}
$$

Denote by $X_{t}^{G}$ the averaging of $X$ with respect to the action of $G$, i.e.,

$$
X_{t}^{G}=\int_{G} \rho_{g *}\left(X_{t}\right) d \mu,
$$

where $d \mu$ is the probabilistic Haar measure on $G$, and $\rho_{g}$ denotes the action of $g \in G$. Then $X_{t}^{G}$ is a $G$-invariant time-dependent vector field. Since $\Pi_{t}$ is invariant under the action of $G$, it follows from Equation (3.10) that we also have

$$
L_{X_{t}^{G}} \Pi_{t}=-\frac{d \Pi_{t}}{d t} .
$$

Denote by $\phi_{t}^{G}$ the flow $X_{t}^{G}$. Then $\phi_{t}^{G}$ commutes with the action of $G$. Equation (3.12) implies that $\phi_{t *}^{G}\left(\Pi_{0}\right)=\Pi_{t}$. In particular, $\phi_{1}^{G}$ is a $G$-equivariant local diffeomorphism such that $\phi_{1 *}^{G}\left(\Pi_{0}\right)=\Pi_{1}=\Pi_{N}+\Pi_{S}$. This concludes the proof of Theorem 1.1.

\section{Equivariant linearization of Poisson structures}

Theorem 4.1. Let $\left(P^{n}, \Pi\right)$ be a smooth Poisson manifold, $p$ a point of $P, 2 r=$ rank $\Pi(p)$, and $G$ a compact Lie group which acts on $P$ in such a way that the action preserves $\Pi$ and fixes the point $p$. Assume that the linear part of transverse Poisson structure of $\Pi$ at $p$ corresponds to a semisimple compact Lie algebra $\mathfrak{k}$. Then there is a smooth canonical local coordinate system $\left(x_{1}, y_{1}, \ldots, x_{r}, y_{r}, z_{1}, \ldots, z_{n-2 r}\right)$ near $p$, in which the Poisson structure $\Pi$ can be written as

$$
\Pi=\sum_{i=1}^{r} \frac{\partial}{\partial x_{i}} \wedge \frac{\partial}{\partial y_{i}}+\frac{1}{2} \sum_{i, j, k} c_{i j}^{k} z_{k} \frac{\partial}{\partial z_{i}} \wedge \frac{\partial}{\partial z_{j}},
$$


where $c_{i j}^{k}$ are structural constants of $\mathfrak{k}$, and in which the action of $G$ is linear and preserves the subspaces $\left\{x_{1}=y_{1}=\ldots x_{r}=y_{r}=0\right\}$ and $\left\{z_{1}=\ldots=z_{n-2 r}=0\right\}$.

Proof. Invoking Theorem 1.1, we may assume that $\Pi$ is already equivariantly splitted, i.e. $\Pi=\sum_{i=1}^{r} \frac{\partial}{\partial x_{i}} \wedge \frac{\partial}{\partial y_{i}}+\sum_{i, j} f_{i j}(z) \frac{\partial}{\partial z_{i}} \wedge \frac{\partial}{\partial z_{j}}$. It remains to linearize the transverse Poisson structure $\Pi_{N}=\sum_{i, j} f_{i j}(z) \frac{\partial}{\partial z_{i}} \wedge \frac{\partial}{\partial z_{j}}$ on $N$ in an equivariant way. But this last step is provided by the following results of Conn and Ginzburg:

Theorem 4.2 (Conn [4]). Any smooth Poisson structure, which vanishes at a point and whose linear part at that point is of semisimple compact type, is locally smoothly linearizable.

Theorem 4.3 (Ginzburg [7]). Assume that a Poisson structure $\Pi$ vanishes at a point $p$ and is smoothly linearizable near $p$. If there is an action of a compact Lie group $G$ which fixes $p$ and preserves $\Pi$, then $\Pi$ and this action of $G$ can be linearized simultaneously.

Indeed, by Theorem 4.2, the transverse Poisson structure $\Pi_{N}$ is smoothly linearizable because its linear part is compact semisimple. As a consequence, by Theorem $4.3, \Pi_{N}$ can be linearized in a $G$-equivariant way.

\section{Appendix}

In this appendix we will give a proof of Proposition 2.4. We will assume that our linear Poisson structure corresponds to a 3 -dimensional Lie algebra $\mathfrak{g}$ (the case of dimension 2 is similar and simpler and can be reduced from the 3 -dimensional case). Recall that any 3 -dimensional Lie algebra $\mathfrak{g}$ over $\mathbb{R}$ belongs to one of the following types:

(1) Solvable: $\mathfrak{g}=\mathbb{R} \ltimes_{A} \mathbb{R}^{2}$ where $A=\left(\begin{array}{ll}a & b \\ c & d\end{array}\right)$ is a 2-by-2 matrix, i.e. with Lie brackets $[x, y]=a y+b z,[x, z]=c y+d z,[y, z]=0$.

(2) Simple: $\mathfrak{s o}(3, \mathbb{R})$ or $\mathfrak{s l}(2, \mathbb{R})$.

We will prove that any vector field $X$ tangent to the symplectic foliation of $\mathfrak{g}^{*}$ (i.e. the foliation by coadjoint orbits on $\mathfrak{g}^{*}$ ) can be expressed as a smooth combination of the Hamiltonian vector fields $X_{x}, X_{y}$ and $X_{z}$, where $(x, y, z)$ is a basis of $\mathfrak{g}$.

Let us first consider the case when $\mathfrak{g}=\mathbb{R} \ltimes_{A} \mathbb{R}^{2}$. In this case, our linear Poisson structure $\Pi$ can be written as:

$$
\Pi=\frac{\partial}{\partial x} \wedge\left((a y+b z) \frac{\partial}{\partial y}+(c y+d z) \frac{\partial}{\partial z}\right) .
$$

We distinguish two subcases.

1) The matrix $A$ has non-zero determinant.

A vector field tangent to the symplectic foliation can be written as $Z=f \frac{\partial}{\partial x}+$ $g\left((a y+b z) \frac{\partial}{\partial y}+(c y+d z) \frac{\partial}{\partial z}\right)$ where the function $f$ vanishes for $(a y+b z, c y+d z)=(0,0)$.

Since the mapping $(x, y, z) \mapsto(x, a y+b z, c y+d z)$ defines new smooth coordinates, we may write $f=(a y+b z) f_{1}+(c y+d z) f_{2}$ for smooth functions $f_{1}$ and $f_{2}$. 
Finally we obtain $Z=f_{1} X_{y}+f_{2} X_{z}-g X_{x}$ for smooth functions $f_{1}, f_{2}$ and $g$ as desired.

2) The determinant of $A$ is equal to zero. In the case $a=b=c=d=0$, the Lie algebra considered is abelian and the Poisson structure is trivial so in this case there is nothing to prove.

In the nontrivial subcase we may write,

$$
\Pi=\frac{\partial}{\partial x} \wedge\left(B \frac{\partial}{\partial y}+\lambda B \frac{\partial}{\partial z}\right)
$$

$B$ being a linear function in $y$ and $z$. After a linear change we may assume that $\Pi=\bar{B} \frac{\partial}{\partial x} \wedge \frac{\partial}{\partial \bar{y}}$.

A vector field tangent to the symplectic foliation is of the form $Z=f \frac{\partial}{\partial x}+g \frac{\partial}{\partial \bar{y}}$ where the functions $f$ and $g$ vanish when $\bar{B}=0$. Since $\bar{B}$ is a non-trivial linear function in $\bar{y}$ and $z$, we may write $f=\bar{B} f_{1}$ and $g=\bar{B} g_{1}$. Therefore we may write $Z=f_{1} X_{x}+g_{1} X_{\bar{y}}$.

Consider now the case when $\mathfrak{g}$ is simple. We will use the following lemma, which is a smooth version of de Rham's division lemma, due to Moussu [12]:

Lemma 5.1. Let $\alpha$ be a smooth (resp. analytic) 1-form on a neighbourhood of the origin in $\mathbb{R}^{n}$ for which the origin is an algebraically isolated singularity, then for any smooth (resp. analytic) p-form $\omega$ such that $\omega \wedge \alpha=0$ we can factorize $\omega$ as $\omega=\beta \wedge \alpha$ for a smooth (resp. analytic) $(p-1)$-form $\beta$.

Denote by $\Pi$ the linear Poisson structure, it can be written as $\Pi=x \frac{\partial}{\partial y} \wedge \frac{\partial}{\partial z}+$ $y \frac{\partial}{\partial z} \wedge \frac{\partial}{\partial x}+z \frac{\partial}{\partial x} \wedge \frac{\partial}{\partial y}$ (in the case of $\left.\mathfrak{s o}(3, \mathbb{K})\right)$ or as $\Pi=z \frac{\partial}{\partial x} \wedge \frac{\partial}{\partial y}+x \frac{\partial}{\partial z} \wedge \frac{\partial}{\partial x}+y \frac{\partial}{\partial y} \wedge \frac{\partial}{\partial z}$ (in the case of $\mathfrak{s l}(2, \mathbb{K}))$.

Let $\Omega$ be the volume form $\Omega=d x \wedge d y \wedge d z$, then the map $\Omega^{b}: \mathcal{V}^{p}\left(\mathfrak{g}^{*}\right) \longrightarrow \Omega^{3-p}\left(\mathfrak{g}^{*}\right)$ from the space of multivector fields to the space of forms defined by $\Omega^{b}(A)=i_{A} \Omega$ is an isomorphism.

Let $X$ be a vector field tangent to the symplectic foliation. The condition of tangency to the symplectic foliation implies the relation $X \wedge \Pi=0$. Under the above linear isomorphism this condition becomes $i_{X} \Omega \wedge i_{\Pi} \Omega=0$. Since $i_{\Pi} \Omega$ has isolated singularities at the origin, we can now apply lemma 5.1 to write $i_{X} \Omega=\beta \wedge i_{\Pi} \Omega$ for a smooth one-form $\beta$.

Finally, we make convenient substitutions to obtain $\left.X=i_{X} \Omega\right\lrcorner\left(\frac{\partial}{\partial x} \wedge \frac{\partial}{\partial y} \wedge \frac{\partial}{\partial z}\right)=$ $\left.\left.\left(\beta \wedge i_{\Pi} \Omega\right)\right\lrcorner\left(\frac{\partial}{\partial x} \wedge \frac{\partial}{\partial y} \wedge \frac{\partial}{\partial z}\right)=\beta\right\lrcorner \Pi$. From this we conclude the proof of proposition 2.4 since from this equality if $\beta=f d x+g d y+h d z$ then $X=f X_{x}+g X_{y}+h X_{z}$ as desired. 


\section{Acknowledgements}

We are indebted to Michèle Vergne for drawing our attention to the paper of Dixmier [5] which contains results about the division property stated in section 2 .

We would like to thank Viktor Ginzburg for his useful comments and suggestions on the problem. We would also like to thank David Martínez-Torres for carefully reading a previous version of this paper and pointing out some misprints.

\section{References}

[1] A. Alekseev and E. Meinrenken, Poisson geometry and the Kashiwara-Vergne conjecture, C. R. Math. Acad. Sci. Paris 335 no.9, (2002) 723-728.

[2] A. Alekseev and E. Meinrenken, Ginzburg-Weinstein via Gelfand-Zeitlin, preprint 2005: math.DG/0506112.

[3] S. Bochner, Compact groups of differentiable transformations, Ann. of Math. (2) 46 (1945) 372-381.

[4] J. Conn, Normal forms for smooth Poisson structures, Ann. of Math. (2) 121 no. 3, (1985) $565-593$.

[5] J. Dixmier, Champs de vecteurs adjoints sur les groupes et algèbres de Lie semi-simples, J. Reine Angew. Math. 309 (1979) 183-190.

[6] J.P. Dufour and N. T. Zung, Poisson Structures and their normal forms, Birkhauser, Progress in Mathematics, 242 (2005).

[7] V. Ginzburg, Momentum mappings and Poisson cohomology, Internat. J. Math. 7 no. 3, (1996) 329-358.

[8] V. Ginzburg and A. Weinstein, Lie-Poisson structure on some Poisson Lie groups, J. Amer. Math. Soc. 5 no. 2, (1992) 445-453.

[9] V. Guillemin, E. Lerman and S. Sternberg, Symplectic fibrations and multiplicity diagrams, Cambridge University Press, Cambridge (1996).

[10] Ph. Monnier and N. T. Zung, Normal forms of vector fields on Poisson manifolds, math.SG/0509144, to appear in Annales Math. Blaise Pascal.

[11] J. Moser, On the volume elements on a manifold, Trans. Amer. Math. Soc. 120 (1965) 286-294.

[12] R. Moussu, Le théorème de de Rham sur la division des formes, C. R. Acad. Sci. Paris Sér. A-B 280 no. 6, (1975) 329-332.

[13] S. Sternberg, Minimal coupling and the symplectic mechanics of a classical particle in the presence of a Yang-Mills field, Proc. Nat. Acad. Sci. U.S.A. 74 no. 12, (1977) 5253-5254.

[14] Y. Vorobjev, Coupling tensors and Poisson geometry near a single symplectic leaf, Lie algebroids and related topics in differential geometry (Warsaw, 2000), 249-274, Banach Center Publ., 54, Polish Acad. Sci., Warsaw (2001).

[15] . Poisson equivalence over a symplectic leaf, Quantum algebras and Poisson geometry in mathematical physics, 241-277, Amer. Math. Soc. Trans. Ser. 2, 216, Amer. Math. Soc., Providence, RI (2005).

[16] A. Weinstein, Lectures on symplectic manifolds, CBMS Regional Conference Series in Mathematics, 29 American Mathematical Society, Providence, RI (1977).

[17] _. The local structure of Poisson manifolds,, J. Differential Geom. 18 (1983), no. 3, 523557.

Laboratoire Emile Picard, UMR 5580 CNRS, Université Toulouse III

E-mail address: miranda@picard.ups-tlse.fr

Laboratoire Emile Picard, UMR 5580 CNRS, Université Toulouse III

E-mail address: tienzung@picard.ups-tlse.fr 